\title{
Deep Learning-based Artificial Intelligence Improves Accuracy of Error-prone Lung Nodules
}

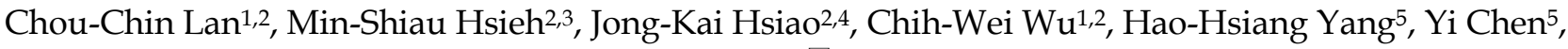 \\ Po-Chun Hsieh6,7, I-Shiang Tzeng, Yao-Kuang Wu ${ }^{1,2}$ \\ 1. Division of Pulmonary Medicine, Taipei Tzu Chi Hospital, Buddhist Tzu Chi Medical Foundation, New Taipei City, Taiwan. \\ 2. School of Medicine, Tzu Chi University, Hualien, Taiwan. \\ 3. Division of thoracic surgery, Taipei Tzu Chi Hospital, Buddhist Tzu Chi Medical Foundation, New Taipei City, Taiwan. \\ 4. Department of Medical Imaging, Taipei TzuChi General Hospital, Buddhist Tzu Chi Medical Foundation, New Taipei City, Taiwan. \\ 5. Department of ASUS Intelligent Cloud Services (AICS), ASUSTek Computer Inc. \\ 6. Department of Chinese Medicine, Taipei Tzu Chi Hospital, Buddhist Tzu Chi Medical Foundation. \\ 7. School of Post-Baccalaureate Chinese Medicine, Tzu Chi University, Hualien, Taiwan. \\ 8. Department of Research, Taipei Tzu Chi Hospital, Buddhist Tzu Chi Medical Foundation, New Taipei City, Taiwan. \\ 9. Department of Research, Taipei Tzu Chi Hospital, Buddhist Tzu Chi Medical Foundation, New Taipei City, Taiwan. \\ $\square$ Corresponding author: Dr. Yao-Kuang Wu, Division of Pulmonary Medicine, Taipei Tzu Chi Hospital, Buddhist Tzu Chi Medical Foundation, New Taipei \\ City, Taiwan. 289 Jianguo Road, Xindian City, Taipei County 23142, Taiwan, Republic of China. Telephone: +886-2-6628-9779 ext. 2259; Fax: +886-2-6628-9009; \\ E-mail: drbfci@yahoo.com.tw.
}

(c) The author(s). This is an open access article distributed under the terms of the Creative Commons Attribution License (https://creativecommons.org/licenses/by/4.0/). See http://ivyspring.com/terms for full terms and conditions.

Received: 2021.11.24; Accepted: 2022.02.22; Published: 2022.03.06

\begin{abstract}
Introduction: Early detection of lung cancer is one way to improve outcomes. Improving the detection of nodules on chest CT scans is important. Previous artificial intelligence (AI) modules show rapid advantages, which improves the performance of detecting lung nodules in some datasets. However, they have a high false-positive (FP) rate. Its effectiveness in clinical practice has not yet been fully proven. We aimed to use $\mathrm{Al}$ assistance in CT scans to decrease FP.

Materials and methods: CT images of 60 patients were obtained. Five senior doctors who were blinded to these cases participated in this study for the detection of lung nodules. Two doctors performed manual detection and labeling of lung nodules without Al assistance. Another three doctors used $\mathrm{Al}$ assistance to detect and label lung nodules before manual interpretation. The $\mathrm{Al}$ program is based on a deep learning framework.

Results: In total, 266 nodules were identified. For doctors without Al assistance, the FP was 0.6170.650 /scan and the sensitivity was $59.2-67.0 \%$. For doctors with Al assistance, the FP was 0.067 to $0.2 /$ scan and the sensitivity was $59.2-77.3 \%$ This Al-assisted program significantly reduced FP. The error-prone characteristics of lung nodules were central locations, ground-glass appearances, and small sizes. The Al-assisted program improved the detection of error-prone nodules.

Conclusions: Detection of lung nodules is important for lung cancer treatment. When facing a large number of CT scans, error-prone nodules are a great challenge for doctors. The Al-assisted program improved the performance of detecting lung nodules, especially for error-prone nodules.
\end{abstract}

Key words: artificial intelligence; lung nodules; CT images

\section{Introduction}

Lung cancer is one of the leading causes of cancer-related deaths worldwide [1]. The prognosis of lung cancer depends largely on the stage of the tumor. Surgical treatment is the only curative treatment for patients with lung cancer [2]. Patients with lung cancer at an operable stage have higher survival rates than those with metastatic disease. Therefore, early detection of early lung cancer is important [1].

The diagnosis and treatment of early stage lung cancer remains challenging. Chest $\mathrm{CT}$ is still the main tool used to diagnose lung cancer [3]. Using chest CT scans to identify lung nodules may help physicians 
find early lung cancer. Many efforts have been made to detect lung nodules on chest CT scans to detect early lung cancer [1]. Improving the diagnosis of lung nodules on chest CT scans may help diagnose early lung cancer and improve prognosis. Early detection of lung nodules may help in early detection of early lung cancer, which might improve the prognosis of lung cancer patients, and reduce medical costs. However, manually detecting a large number of CT scans is a great burden, requires attention, and is very timeconsuming, making it prone to errors. Moreover, lung nodules are sometimes very difficult to detect, even for experienced doctors.

Artificial intelligence (AI) has rapid advantages and exciting achievements in imaging diagnosis. Therefore, many studies have used AI for the detection of lung nodules. These efforts have attempted to improve the accuracy of the detection of lung nodules [1]. AI applications have great potential for improving the diagnosis of lung nodules on CT scans. Many of the programs performed well in detecting lung nodules in certain datasets. However, its effectiveness in clinical practice has not been fully proven [1]. The low sensitivity or high false-positive rate limits its practical application in clinical practice [1]. Therefore, more research is needed to study clinical AI applications.

Therefore, our current research is aimed at using AI-based computer-aided diagnostic systems to help clinicians detect lung nodules on CT scans.

\section{Materials and methods}

\section{$C T$ acquisition and reading}

CT scan images of 60 cases were obtained for the detection of lung nodules. The chest CT scan was performed using a 64-slice detector, GE LightSpeed, and the thickness of the lung window slice was 2.5 $\mathrm{mm}$. Five senior doctors (all had more than 10 years of experience in reading chest $\mathrm{CT}$ scans), including three chest physicians, one chest surgeon, and one radiologist, participated in this study for the detection of lung nodules. All of the doctors were blinded to all these cases. Two doctors (doctors 1 and 2) performed manual detection and labeling of lung nodules without AI assistance as a traditional method. Another three doctors (doctors 3, 4, and 5) received AI assistance to detect and label lung nodules before manual interpretation. The study was approved by the Taipei Tzu Chi Hospital, Buddhist Tzu Chi Medical Foundation Institutional Review Board (Protocol Number: 09-X-007).

\section{Setting and Notations of Al algorithm}

$$
\mathbb{H}=\left\{H_{i}=\left(p_{i}, d_{i}\right)\right\}_{i=1}^{N}
$$

$$
\begin{gathered}
p_{i}=\left(x_{i}, y_{i}, z_{i}\right) \in \mathbb{R}^{3} d_{i} \\
\mathbb{H} \\
y \in\{0,1\}
\end{gathered}
$$

Given a lung 3D CT scan image $I$ with $N$ nodules, we denote the set of nodules, while $\left\{\hat{z}_{1}, \hat{z}_{2}, \ldots, \hat{z}_{n}, n \leq\right.$ $k$ \} denotes the spatial location and diameter of the $i$ th nodule. Weakly supervised pulmonary nodule detection is not accessible during training. Instead, one typically observes the image label from electronic medical records (EMR) during the training stage, which indicates whether the CT scan contains nodules. In our work, we further consider auxiliary information from EMR, including the number $k$ of nodules and the slice indices of each nodule on CT scan.

Fig. 1 shows our proposed deep learning framework for weakly supervised pulmonary nodule detection. As shown in Fig. 1, a $\widehat{\mathbb{H}}=\left\{\widehat{H}_{i}=\left(\hat{p}_{i}, \hat{d}_{i}\right)\right\}_{i=1}^{M}$ pre-trained nodule of the 3D feature pyramid network (3D-FPN) [1] is applied to extract the preliminary prediction (i.e., features, bounding box location) of each nodule. Such prediction outputs can be viewed as primitive nodule proposals, and the aforementioned weak EMR labels (i.e., image label $y$, nodule number $k$, and nodule slice index $z$ ) were further utilized to guide the learning of our framework.

\section{Pulmonary nodule detection with supervision}

Previously, multiple instance learning (MIL) [4] has been applied to address object detection in weakly supervised settings, which is realized by observing only image-level labels during training. Without the need to collect any instance-level labels, the above model aims to estimate nodule proposals $\widehat{\mathbb{H}}$, which would be properly associated with the image-level label $y$. For each proposal $\widehat{H}_{i}$, the pooling operation is applied to extract the corresponding feature maps from the 3D-FPN backbone detector, denoted as $\widehat{\mathbb{F}}=\left\{\hat{f}_{i}\right\}_{i=1}^{M}$. In a previous study [5], fully connected layers with a rectified linear unit (ReLU) activation function were deployed to infer the confidence score of each object proposal. Finally, to match the groundtruth image-level prediction, a number of techniques have been proposed to process the predicted $\hat{y}$ from $\widehat{\mathbb{H}}$ [6-8]. In our work, we followed and considered the maximum operator as the MIL pooling function:

$$
\hat{y}=\max \left\{\hat{h}_{i}\right\}_{i=1}^{M}=\max \left\{\operatorname{MIL}\left(\hat{f}_{i}\right)\right\}_{i=1}^{M}
$$

where MIL denotes the MIL branch, and $\hat{h}_{i}$ is the predicted score of the proposals in the proposed learning framework. We noted that we fed the extracted visual features into our weakly supervised 
pulmonary nodule detection module without adjusting the weights of the original ResNet- 18 or FPN backbones. This allowed us to focus on the network modules for predicting and re-ranking the extracted nodule proposals under different weak supervision.

The data used for pre-training ResNet 18 comed from the lung nodule open dataset of Lung Image Database Consortium image collection (LIDC-IDRI) (https://wiki.cancerimagingarchive.net/plugins/ servlet/mobile?contentId=1966254\#content/view / 1966254), including 1018 CT volumes from 1010 different patients. A range of scanner manufacturers and models was represented (670 scans from seven different GE Medical Systems LightSpeed scanner models, 74 scans from four different Philips Brilliance scanner models, 205 scans from five different Siemens Definition, Emotion, and Sensation scanner models, and 69 scans from Toshiba Aquilion scanner) [9]. After training, we tested the model performance on hospital private data, including $60 \mathrm{CT}$ volumes from 60 patients.

\section{Reference standard}

The lung nodules interpreted by AI and most doctors (at least three doctors) were used as standard references. Sensitivity refers to the rate at which the physician has labeled, and most other physicians and AI are also labeled. Lung nodules labeled by the expert, but beyond the consensus of most other experts, are regarded as false positives (FP) [10].

\section{Analysis}

The overall sensitivity and FP were analyzed. The influence of nodular location (upper, middle, lower; central or peripheral), size, and texture in the CT scan was analyzed.

\section{Results}

\section{Demographic characteristics}

The demographic data of the patients are summarized in Table 1 . The mean age was $62.6 \pm 11.0$ years. The mean body height was $159.8 \pm 8.8 \mathrm{~cm}$ and the mean body weight was $61.1 \pm 13.6 \mathrm{~kg}$. Among them, 27 were males $(45 \%)$ and 33 were females $(55 \%)$. Most patients did not smoke $(\mathrm{N}=44,73.3 \%)$, there were $4(6.7 \%)$ current smokers and $12(20.0 \%)$ former smokers.

\section{Overall nodular detection}

There were 266 nodules in 60 patients (Fig. 2). For doctors without AI assistance, the FP was $0.617-0.650 /$ scan (mean $0.634,95 \%$ CI $0.586-0.680$ ) and the sensitivity was $59.2-67.0 \%$ (mean $63.1 \%$, 95\% CI 52.0-74.1\%). For doctors with AI assistance, the FP was 0.067 to $0.2 /$ scan (mean 0.122, 95\% CI $0.000-0.261$ ), and the sensitivity was $59.2-77.3 \%$ (mean $69.8 \%, 95 \%$ CI $50.9-88.6 \%$ ).

Table 1. Demographic characteristics of the patients

\begin{tabular}{lll}
\hline Characteristics & & \\
\hline Age $(\mathrm{yrs})$ & & $62.6 \pm 11.0$ \\
$\mathrm{BH}(\mathrm{cm})$ & & $159.8 \pm 8.8$ \\
$\mathrm{BW}(\mathrm{Kg})$ & Male & $61.1 \pm 13.6$ \\
Gender & Female & $27(45 \%)$ \\
& Non-smoking & $33(55 \%)$ \\
Smoking & Current smoker & $44(73.3 \%)$ \\
& Ex-smoker & $4(6.7 \%)$ \\
& & $12(20.0 \%)$ \\
\hline
\end{tabular}

Abbreviations: $\mathrm{BH}$, body height; BW, body weight.

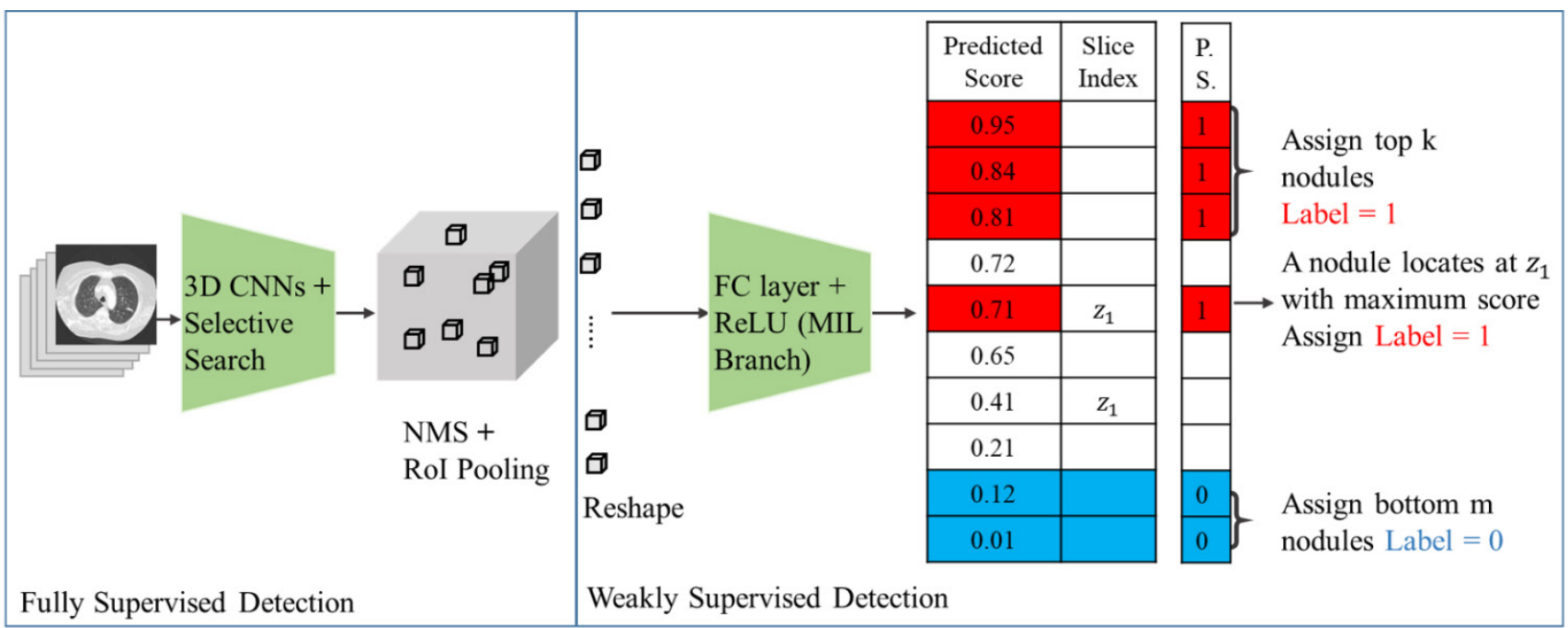

Figure 1. Framework for pulmonary nodule detection. The 3D CNN is a pre-trained fully supervised detector that serves as the detector backbone to extract nodule proposals and features in weakly supervised settings. In addition to image-level labels to predict the pseudo labels for each proposal, this model additionally observed nodule numbers and slice index information from EMR to guide the learning process. Abbreviations: 3D CNN: 3-Dimentional convolutional neural network; NMS, non-maximum suppression; Rol pooling, region of interest pooling; FC layer, fully connected layer; ReLU, rectified linear unit; MIL, multiple instance learning; P.S., pseudo labels. 


\begin{tabular}{|c|c|}
\hline & $\begin{array}{c}\text { Number of } \\
\text { Nodules }\end{array}$ \\
\hline Total & 266 \\
\hline
\end{tabular}

\begin{tabular}{|l|c|c|} 
& $\begin{array}{c}\text { FP/sca } \\
\text { n }\end{array}$ & Sensitivity \\
\hline Doctor 1 & 0.650 & $59.2 \%$ \\
\hline Doctor 2 & 0.617 & $67.0 \%$ \\
\hline Doctor 3 & 0.067 & $59.2 \%$ \\
\hline Doctor 4 & 0.100 & $72.8 \%$ \\
\hline Doctor 5 & 0.200 & $77.3 \%$ \\
\hline
\end{tabular}

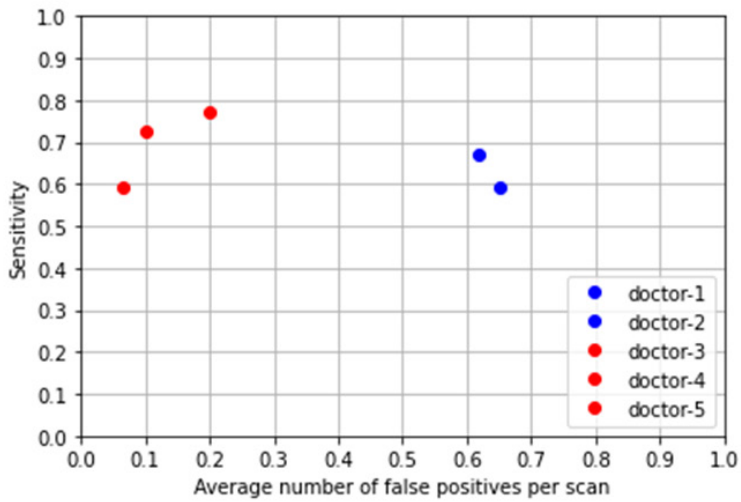

Figure 2. Overall nodular detection.

A

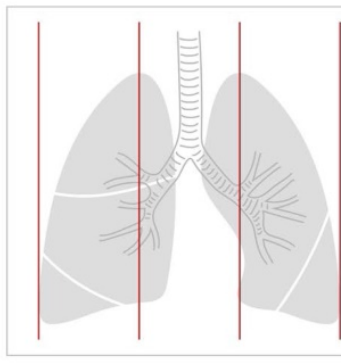

\begin{tabular}{|c|c|}
\hline Left & $\begin{array}{c}\text { Number of } \\
\text { Nodules }\end{array}$ \\
\hline Center & 60 \\
\hline Right & 150 \\
\hline Total & 56 \\
\hline
\end{tabular}

B

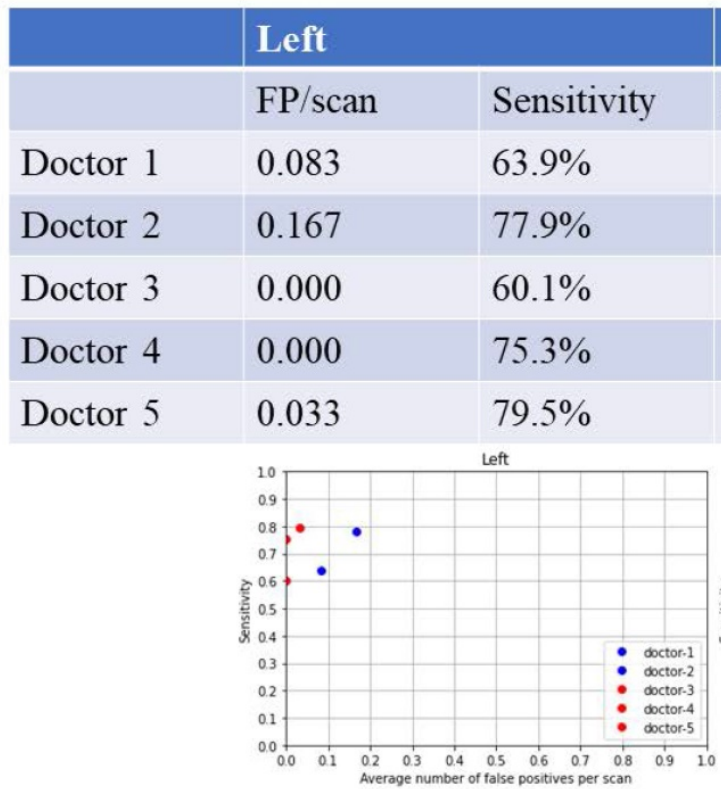

Center

$\mathrm{FP} /$ scan

0.400

0.267

0.067

0.100

0.117

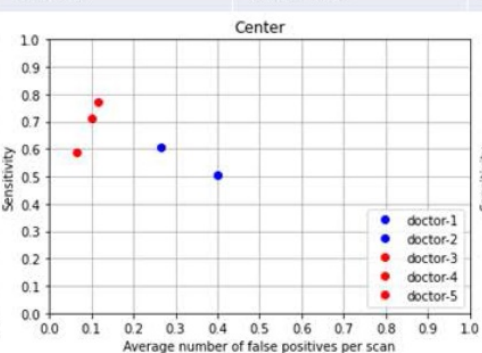

\section{Right}

Sensitivity $\mathrm{FP} / \mathrm{scan}$

0.167

0.183

0.000

0.000

0.050

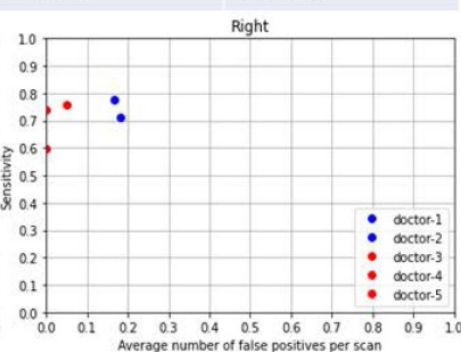

Figure 3. Left, central and right lung fields and nodular detection. Number of pulmonary nodular detection in left, center and right lung fields. False positive and sensitivity of nodular detection in left, center and right lung fields.

\section{Left, central and right lung fields and nodular detection}

There were 60 nodules in the left lung fields, 150 nodules in the central lung fields, and 56 nodules in the right lung fields (Fig. 3A). The summary of FP and sensitivity for detecting lung nodules in left, central and right lung fields are shown in Table 2 . For the left lung field, the FP was $0.083-0.167 /$ scan (mean 0.125, $95 \%$ CI $0.006-0.243$ ) and the sensitivity was $63.9-77.9 \%$ (mean 70.9\%, 95\% CI 51.1-90.6\%) without AI assistance (Fig. 3B). With AI assistance, the FP was 
$0-0.033$ / scan (mean $0.011,95 \%$ CI $0.000-0.049$ ), and the sensitivity was $60.1-75.9 \%$ (mean $71.6 \%$, 95\% CI $51.2-92.1 \%)$. For the central lung fields, the FP was $0.267-0.40 /$ scan (mean $0.334,95 \%$ CI $0.316-0.352$ ) and the sensitivity was $50.3-60.8 \%$ (mean $55.6 \%$, 95\% CI 40.7-70.3\%) without AI assistance. With AI assistance, the FP was $0.067-0.117 /$ scan (mean $0.094,95 \%$ CI $0.043-0.145$ ) and the sensitivity was $58.6-76.9 \%$ (mean $68.9 \%, 95 \%$ CI $50.1-87.6 \%)$. For the right lung field, the FP was 0.167-0.183/scan (mean 0.176, 95\% CI $0.153-0.197$ ) and the sensitivity was $71.2-77.7 \%$ (mean $74.5 \%, 95 \%$ CI $65.2-83.6 \%$ ) without AI assistance. With AI assistance, the FP was 0-0.05/scan (mean 0.017, 95\% CI 0.000-0.074) and the sensitivity was $59.8-75.6 \%$ (mean 69.7\%, 95\% CI 52.4-87.0\%).

\section{Upper, middle and lower lung fields and nodular detection}

There were 83 nodules in the upper lung fields, 123 nodules in the middle lung fields, and 50 nodules in the lower lung fields (Fig. 4A). The summary of FP and sensitivity for detecting lung nodules in upper, middle and lower lung fields are shown in Table 2.
For the upper lung fields, the FP was $0.100-0.183$ / scan (mean $0.142,95 \%$ CI $0.024-0.258$ ) and the sensitivity was $66.5-73.0 \%$ (mean $69.8 \%, 95 \%$ CI $60.5-78.9 \%$ ) of doctors without AI assistance and FP was $0.033-0.083$ /scan (mean 0.055, 95\% CI 0.004-0.106), and the sensitivity was $68.3-84.5 \%$ (mean $77.6 \%, 95 \%$ CI 60.8-94.2\%) for doctors with AI assistance (Fig. 4B). For the middle lung fields, the FP was $0.267-0.333$ / scan (mean 0.300, 95\% CI 0.206-0.393) and the sensitivity was $52.0-65.0 \%$ (mean $58.5 \%$, 95\% CI 40.1-76.8\%) of doctors without $\mathrm{AI}$ assistance and FP was $0.017-0.050 /$ scan (mean $0.033,95 \%$ CI $0.003-0.066$ ) and the sensitivity was $53.9-75.5 \%$ (mean $66.1 \%$, 95\% CI $43.9-88.2 \%$ ) of doctors with AI assistance. For the lower lung fields, the FP was $0.1-0.267 /$ scan (mean $0.184,95 \%$ CI $0.000-0.419)$ and the sensitivity was $45.2-43.9 \%$ (mean $44.5 \%$, 95\% CI $42.7-46.3 \%$ ) of doctors without AI assistance and FP was $0.017-0.033$ /scan (mean 0.017, 95\% CI 0.000-0.049) and the sensitivity was $37.4-51.2 \%$ (mean $45.4 \%$, 95\% CI $31.0-59.7 \%$ ) of doctors with AI assistance.

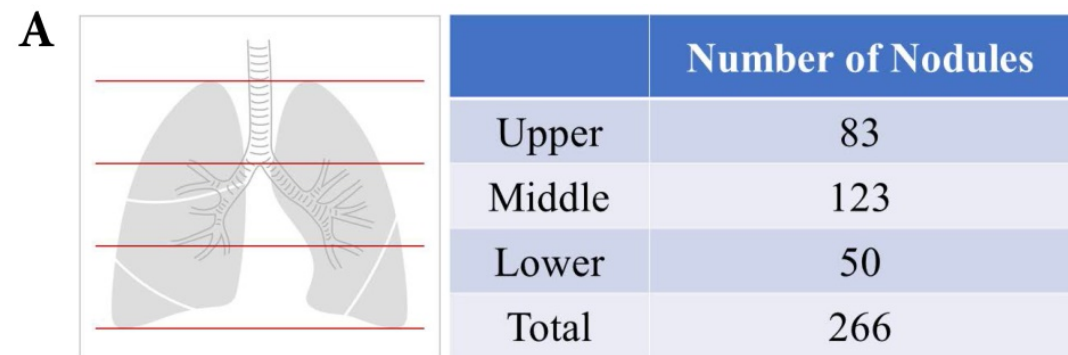

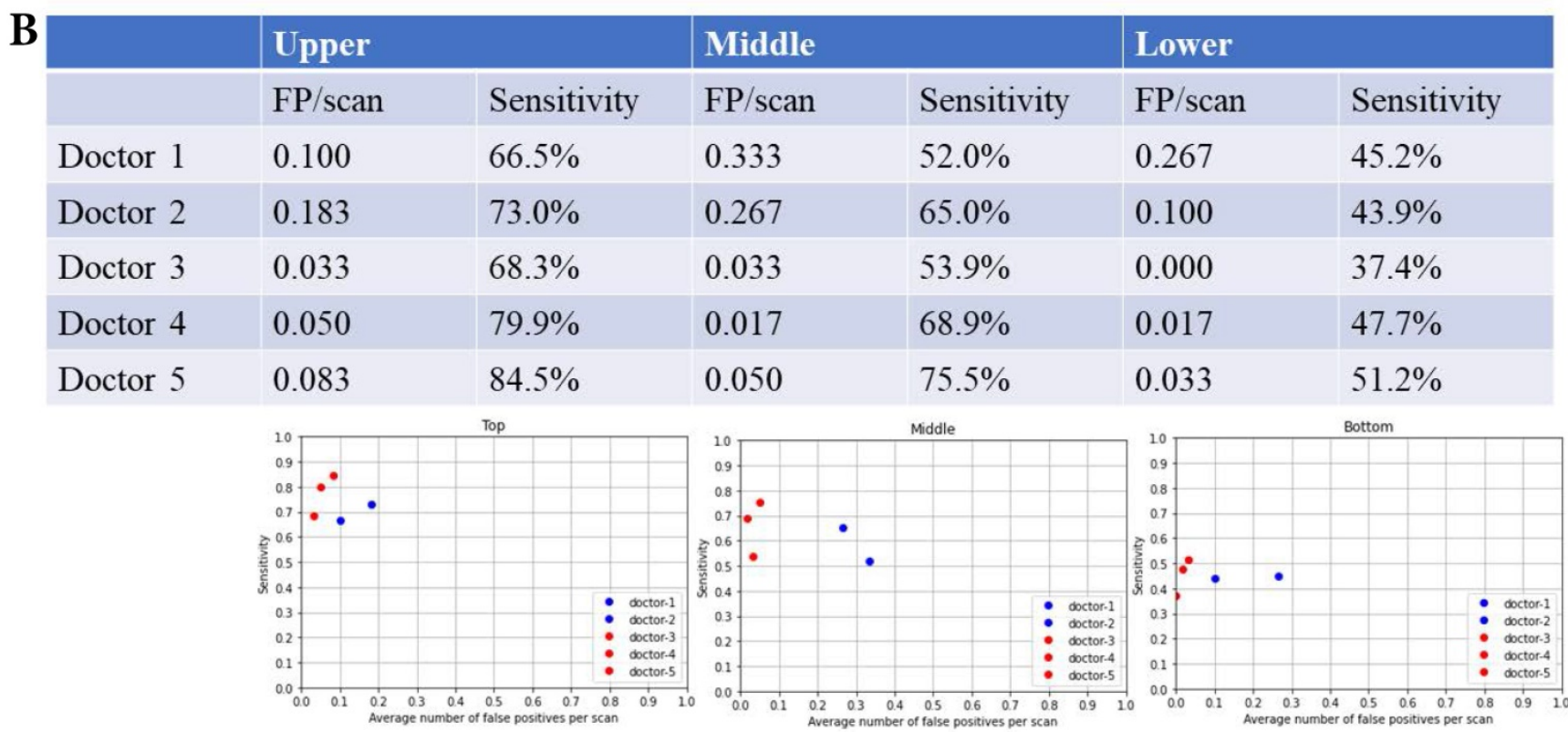

Figure 4. Upper, middle and lower lung fields and nodular detection. (A) Number of pulmonary noular detection in upper, milddle and lower lung fields. (B) False positive and sensitivity of nodular detection in upper, milddle and lower lung fields. 
Table 2. The false positive and sensitivity of Al detection in different location, size and texture

\begin{tabular}{|c|c|c|c|c|c|c|c|c|c|c|c|c|c|}
\hline & & \multicolumn{6}{|c|}{ Location } & \multicolumn{6}{|c|}{ Location } \\
\hline & & \begin{tabular}{|l|} 
Left \\
FP/scan
\end{tabular} & Sensitivity & $\begin{array}{l}\text { Central } \\
\mathrm{FP} / \text { scan }\end{array}$ & Sensitivity & \begin{tabular}{|l} 
Right \\
FP/scan
\end{tabular} & Sensitivity & \begin{tabular}{|l|} 
Upper \\
$\mathrm{FP} /$ scan
\end{tabular} & Sensitivity & \begin{tabular}{|l} 
Middle \\
FP/scan
\end{tabular} & Sensitivity & \begin{tabular}{|l} 
Lower \\
$\mathrm{FP} /$ scan
\end{tabular} & Sensitivity \\
\hline \multirow[t]{3}{*}{ without AI } & mean & 0.125 & $70.9 \%$ & 0.334 & $55.6 \%$ & 0.176 & $74.5 \%$ & 0.142 & $69.8 \%$ & 0.300 & $58.5 \%$ & 0.184 & $44.5 \%$ \\
\hline & lower $95 \% \mathrm{CI}$ & 0.006 & $51.1 \%$ & 0.316 & $40.7 \%$ & 0.153 & $65.2 \%$ & 0.024 & $60.5 \%$ & 0.206 & $40.1 \%$ & 0.000 & $42.7 \%$ \\
\hline & upper $95 \%$ CI & 0.243 & $90.6 \%$ & 0.352 & $70.3 \%$ & 0.197 & $83.6 \%$ & 0.258 & $78.9 \%$ & 0.393 & $76.8 \%$ & 0.419 & $46.3 \%$ \\
\hline \multirow[t]{5}{*}{ with AI } & mean & 0.011 & $71.6 \%$ & 0.094 & $68.9 \%$ & 0.017 & $69.7 \%$ & 0.055 & $77.6 \%$ & 0.033 & $66.1 \%$ & 0.017 & $45.4 \%$ \\
\hline & lower $95 \%$ CI & 0.000 & $51.2 \%$ & 0.043 & $50.1 \%$ & 0.000 & $52.4 \%$ & 0.004 & $60.8 \%$ & 0.003 & $43.9 \%$ & 0.000 & $31.0 \%$ \\
\hline & upper $95 \%$ CI & 0.049 & $92.1 \%$ & 0.145 & $87.6 \%$ & 0.074 & $87.0 \%$ & 0.106 & $94.2 \%$ & 0.066 & $88.2 \%$ & 0.049 & $59.7 \%$ \\
\hline & & \multicolumn{6}{|c|}{ Size } & \multicolumn{6}{|c|}{ Texture } \\
\hline & & \begin{tabular}{|l|} 
Small \\
FP/scan
\end{tabular} & Sensitivity & \begin{tabular}{|l|} 
Middle \\
FP/scan
\end{tabular} & Sensitivity & \begin{tabular}{|l} 
Large \\
FP/scan
\end{tabular} & Sensitivity & \begin{tabular}{|l|} 
GGO \\
FP/scan
\end{tabular} & Sensitivity & \begin{tabular}{|l|} 
Partial so \\
$\mathrm{FP} /$ scan
\end{tabular} & $\begin{array}{l}\text { lid } \\
\text { Sensitivity }\end{array}$ & \begin{tabular}{|l|} 
solid \\
FP/scan
\end{tabular} & Sensitivity \\
\hline \multirow[t]{3}{*}{ without AI } & mean & 0 & $3.2 \%$ & 0.192 & $69.4 \%$ & 0.442 & $80.2 \%$ & 0.409 & $65.1 \%$ & 0.100 & $74.6 \%$ & 0.125 & $57.9 \%$ \\
\hline & lower $95 \% \mathrm{CI}$ & 0 & $3.2 \%$ & 0.000 & $40.3 \%$ & 0.041 & $77.4 \%$ & 0.101 & $64.7 \%$ & 0.000 & $61.3 \%$ & 0.102 & $39.5 \%$ \\
\hline & upper $95 \% \mathrm{CI}$ & 0 & $3.2 \%$ & 0.545 & $98.3 \%$ & 0.841 & $82.8 \%$ & 0.715 & $65.5 \%$ & 0.334 & $87.8 \%$ & 0.147 & $76.2 \%$ \\
\hline \multirow[t]{3}{*}{ with AI } & mean & 0.077 & $76.3 \%$ & 0.011 & $80.3 \%$ & 0.033 & $59.3 \%$ & 0.039 & $61.5 \%$ & 0.056 & $74.5 \%$ & 0.078 & $74.7 \%$ \\
\hline & lower $95 \%$ CI & 0.000 & $57.5 \%$ & 0.000 & $67.3 \%$ & 0.000 & $35.3 \%$ & 0.000 & $43.6 \%$ & 0.000 & $63.6 \%$ & 0.000 & $51.6 \%$ \\
\hline & upper $95 \%$ CI & 0.177 & $94.9 \%$ & 0.030 & $93.3 \%$ & 0.066 & $83.3 \%$ & 0.108 & $79.3 \%$ & 0.219 & $85.2 \%$ & 0.173 & $97.7 \%$ \\
\hline
\end{tabular}

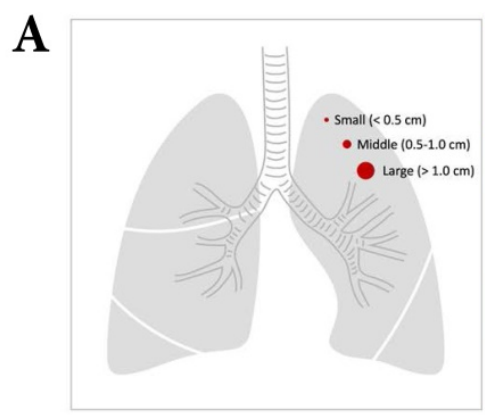

\begin{tabular}{|c|c|}
\hline Small $(<0.5 \mathrm{~cm})$ & $\begin{array}{c}\text { Number of } \\
\text { Nodules }\end{array}$ \\
\hline Middle $(0.5-1.0 \mathrm{~cm})$ & 43 \\
\hline Large $(>1.0 \mathrm{~cm})$ & 94 \\
\hline Total & 129 \\
\hline
\end{tabular}

B

\begin{tabular}{|l|l|l|l|l|l|l|}
\hline & Small & & Middle & & Large & \\
\hline FP/scan & Sensitivity & FP/scan & Sensitivity & FP/scan & Sensitivity \\
\hline Doctor 1 & 0.000 & $3.2 \%$ & 0.067 & $59.1 \%$ & 0.583 & $81.1 \%$ \\
\hline Doctor 2 & 0.000 & $3.2 \%$ & 0.317 & $79.6 \%$ & 0.300 & $79.2 \%$ \\
\hline Doctor 3 & 0.030 & $67.6 \%$ & 0.000 & $72.9 \%$ & 0.033 & $45.8 \%$ \\
\hline Doctor 5 & 0.070 & $75.0 \%$ & 0.017 & $83.1 \%$ & 0.017 & $63.5 \%$ \\
\hline
\end{tabular}
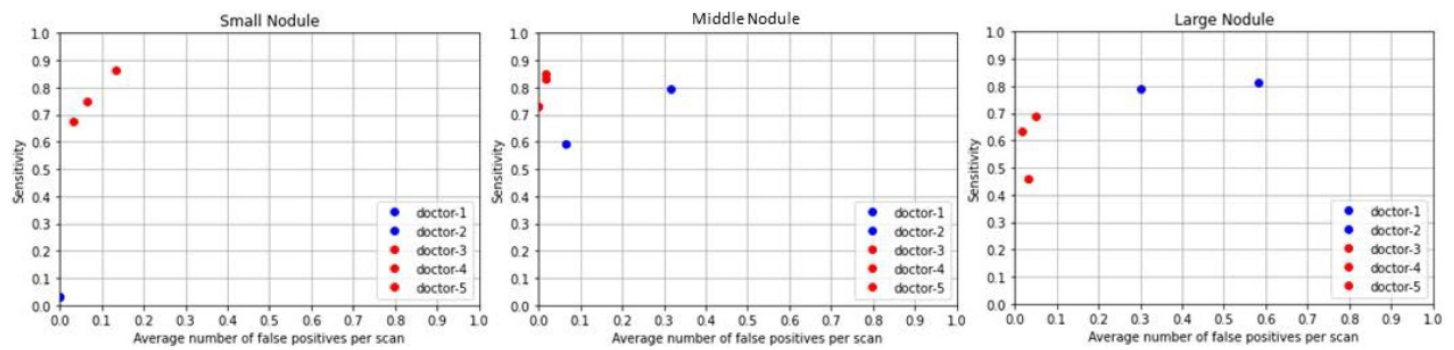

Figure 5. Nodular size and nodular detection. (A) Number of difference sizes of pulmonary nodules. (B) False positive and sensitivity of nodular detection in difference sizes of pulmonary nodules.

\section{Nodular size and nodular detection}

There were 43 small nodules (diameter $<0.5 \mathrm{~cm}$ ), 94 middle nodules (diameter $0.5-1.0 \mathrm{~cm}$ ), and 129 large nodules (diameter $>1.0 \mathrm{~cm}$ ) (Fig. 5A). The summary of FP and sensitivity for detecting lung nodules of different nodular sizes is presented in
Table 2. For the small nodules, the FP was 0 (mean 0 , 95\% CI $0-0$ ) and the sensitivity was 3.2\% (mean 3.2\%, 95\% CI 3.2-3.2\%) of doctors without AI assistance and FP was 0.003-0.13/scan (mean 0.077, 95\% CI $0.000-0.177$ ), and the sensitivity was $64.6-86.2 \%$ (mean $76.3 \%$, 95\% CI $57.5-94.9 \%)$ of doctors with AI 
assistance (Fig. 5B). For the middle nodules, the FP was $0.067-0.317 /$ scan (mean $0.192,95 \%$ CI $0.000-0.545$ ) and the sensitivity was $59.1-79.6 \%$ (mean $69.4 \%, 95 \%$ CI 40.3-98.3\%) of doctors without AI assistance and FP was $0-0.017 /$ scan (mean $0.011,95 \%$ CI $0.000-0.030$ ), and the sensitivity was $72.9-85.0 \%$ (mean $80.3 \%$, 95\% CI 67.3-93.3\%) for doctors with AI assistance. For the large nodules, the FP was 0.3-0.583/scan (mean 0.442, 95\% CI 0.041-0.841) and the sensitivity was 79.2-81.1\% (mean $80.2 \%, 95 \%$ CI 77.4-82.8\%) of doctors without AI assistance and FP was $0.033-0.050 /$ scan (mean $0.033,95 \%$ CI $0.000-0.066$ ) and the sensitivity was $45.8-68.7 \%$ (mean 59.3\%, 95\% CI $35.3-83.3 \%$ ) of doctors with AI assistance.

\section{Nodular texture and nodular detection}

There were 109 ground-glass organization (GGO) nodules, 41 partial nodules, and 116 solid nodules (Fig. 6A). Table 2 summarizes the FP and sensitivity for detecting lung nodules of different nodular textures. For the GGO nodules, the FP was
$0.3-0.517 /$ scan (mean $0.409,95 \%$ CI $0.101-0.715$ ) and the sensitivity was $65.0-65.3 \%$ (mean $65.1 \%, 95 \%$ CI $64.7-65.5 \%$ ) of doctors without AI assistance and FP was $0-0.067 /$ scan (mean $0.039,95 \%$ CI $0.000-0.108$ ) and the sensitivity was $52.2-70.0 \%$ (mean $61.5 \%, 95 \%$ CI 43.6-79.3\%) of doctors with AI assistance (Fig. 6B). For the partial solid nodules, the FP was $0.017-0.183$ /scan (mean $0.100,95 \%$ CI $0.000-0.334$ ) and the sensitivity was $69.9-79.3 \%$ (mean $74.6 \%, 95 \%$ CI $61.3-87.8 \%$ ) of doctors without AI assistance and FP was $0-0.150$ (mean $0.056,95 \%$ CI $0.000-0.219$ ) and the sensitivity was $68.3-78.4 \%$ (mean $74.5 \%$, 95\% CI 63.6-85.2\%) of doctors with AI assistance. For the solid nodules, the FP was 0.117-0.133/scan (mean 0.125, 95\% CI 0.102-0.147) and the sensitivity was 51.4-64.4\% (mean 57.9\%, 95\% CI 39.5-76.2\%) of doctors without AI assistance and FP was 0.050-0.133/scan (mean $0.078,95 \%$ CI $0.000-0.173$ ), and the sensitivity was $51.2-63.5 \%$ (mean $74.7 \%$, 95\% CI $51.6-97.7 \%$ ) of doctors with AI assistance.
A

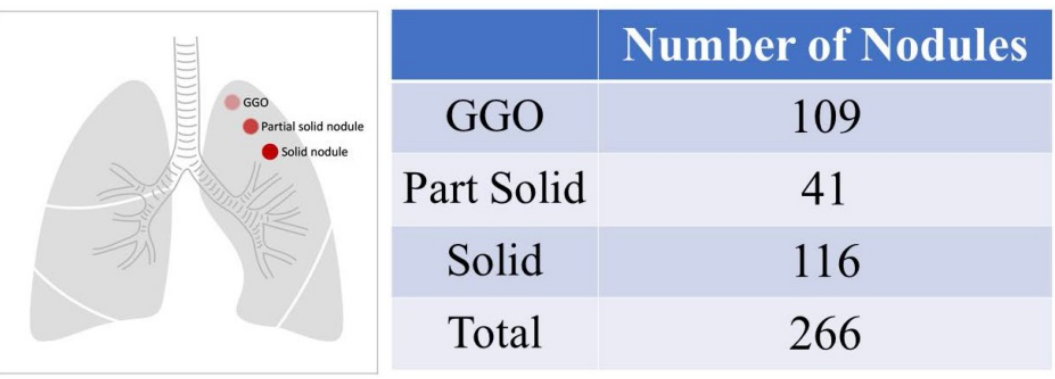

B

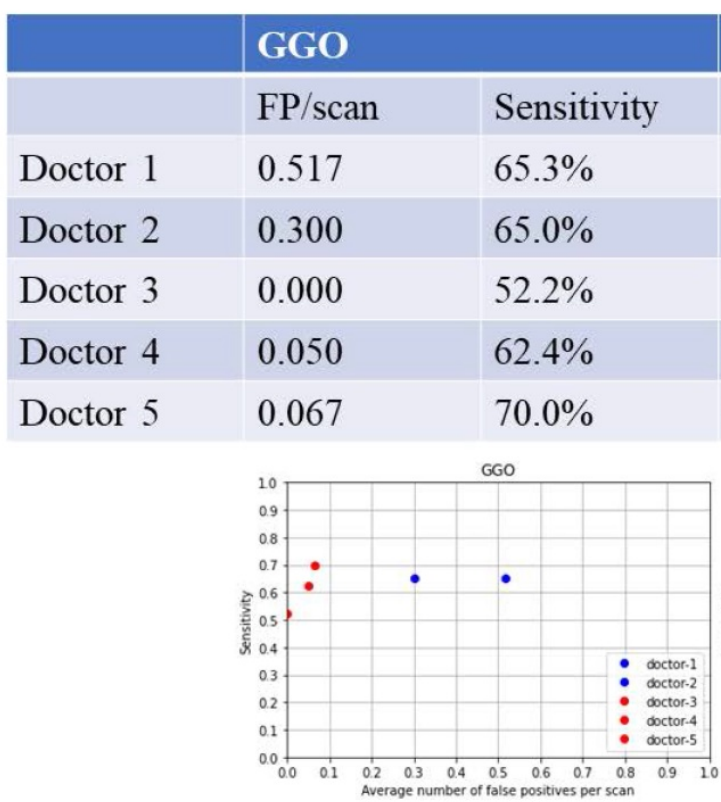

Part Solid

$\mathrm{FP} /$ scan

0.017

0.183

0.017

0.000

0.150

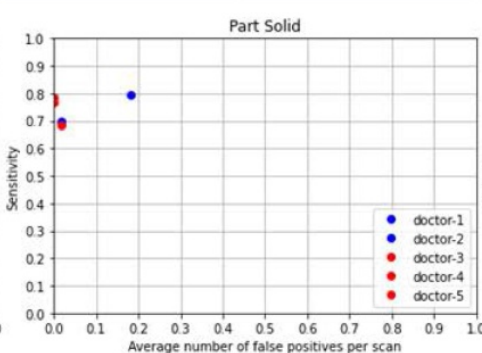

\section{Solid}

Sensitivity $\mathrm{FP} /$ scan

0.117

0.133

0.050

0.050

0.133

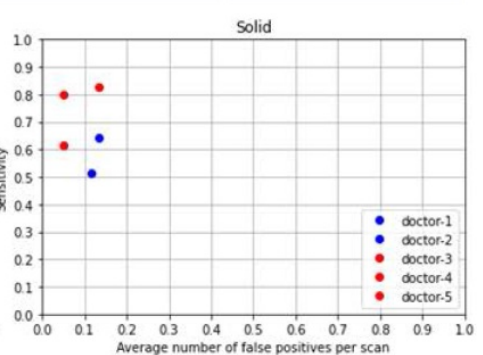

Figure 6. Nodular texture and nodular detection. Number of difference textures of pulmonary nodules. False positive and sensitivity of nodular detection in difference textures of pulmonary nodules. 


\section{Discussion}

The current study has several important findings. Doctors who are not assisted by AI are more likely to have more FP and less sensitivity in their predictions with respect to the center and middle positions. Facing small lung nodules (less than 0.5 $\mathrm{cm})$, the doctor's sensitivity is quite poor. In terms of nodule texture, doctors were more likely to have more FP for GGOs. With the assistance of AI, the overall false positive and sensitivity of the doctor's interpretation can be improved. The accuracy of the above-prone areas or features can also be improved.

A reasonable idea is that if the nodule is too small, the doctors or the AI may easily miss the lung nodules. Previous studies have found that the most common cause of missed diagnosis on CT scans is its small size [11]. Del Ciello et al. suggested that a small diameter $(<7 \mathrm{~mm})$ is one of the causes of failed diagnosis [11]. In our study, the tiny nodules $(<0.5$ $\mathrm{cm}$ ) were quite difficult to manually detect in chest CT scans with a sensitivity of only $3.2 \%$. With AI assistance, the sensitivity was greatly improved for tiny nodules. Several studies have also demonstrated that $\mathrm{AI}$, as a second reader, significantly increases sensitivity in the identification of lung nodules [12].

One of the factors leading to missed lung nodules is their central location [11]. Del Ciello et al. reported that the missing rate of lung nodules in the central area is disproportionately high [11]. Deveraj et al. also found that hilar nodules, that are blind spots on CT scans, are one of the causes of missed lung cancers [13]. Some normal tissues have similar appearances as nodules on CT images. There are more normal lung tissues in the central area, especially near the hilar region. It is sometimes challenging for doctors to distinguish lung nodules from pulmonary vessels, bones, and other structures [11]. This will cause difficulties in interpretation, resulting in a decrease in accuracy. When lung tissues are regarded as lung nodules, they cause an increase in FP. Conversely, if the existing lung nodules are regarded as lung tissues, the sensitivity will be reduced. The approach for differentiating between the tissues and nodules is therefore crucial to reduce FP in an automatic lung nodule detection scheme [1]. In our AI assistant programs, the FP rates were greatly decreased.

The characteristics of lung nodules are also a factor for detecting lung nodules on CT scans. Previously, Li et al. revealed that lung cancers missed on CT screenings are very subtle and appear as small faint nodules [14]. Del Ciello et al. also showed that blurred and unclear margins are also factors of missed lung nodules [11]. Benzakoun et al. also suggested that the ground-glass component may hinder software detection of attenuation differences with the surrounding parenchyma [15]. They reported that partially solid nodules with a sensitivity of $72 \%$ were much better than pure ground-glass nodules with a sensitivity of $28 \%$ [15].

Compared with previous studies, our study showed a balance between sensitivity and FP. Although many previous studies have shown high identification sensitivity, their FP rates are also quite high. Cui et al. built on a 50-layer deep neural network and trained a large multi-center database; its deep learning algorithm showed a sensitivity of $91.0 \%$ but had 2FPs/case [10]. The computer-aided detection algorithms proposed by Ali et al. showed an overall accuracy of $64.4 \%$ (sensitivity $58.9 \%$, specificity $55.3 \%$, PPV 54.2\%, and NPV 60.0\%) [16]. Cao et al. produced a sensitivity of $90 \%$ and FP $1 /$ scan on their 3-Dimentional convolutional neural network (3D-CNN) [17]. Dou et al. also used 3D CNNs and showed a sensitivity of $90.7 \%$ and 4 FPs/case [18] which was similar to that of Setio et al., who used multiview convolutional networks to obtain a sensitivity of $90.1 \%$ and $4 \mathrm{FPs} /$ case [19]. These results showed high sensitivity, but their FP rates were also quite high at approximately 2-4 FPs/scan $[10,16,17$, 19].

The balance between the sensitivity and FP is important. Most previous models showed high sensitivity and high FP in CT screening of lung nodules using computer-aided detection. Modern technologies allow doctors to detect focal lung lesions more efficiently. However, FP is a critical problem in lung nodule detection because FP results in unnecessary follow-up tests and expenditures [16]. It also leads to increased patient suffering and even unnecessary invasive procedures to confirm the diagnosis. This results in an increased risk of procedures. In addition, frequent and regular follow-up of chest CT scans may also lead to radiation-induced cancer [20]. Previous studies also showed that computer-aided detection software shows high FP, which represents a major limitation in the wider use of the system [11]. Therefore, most computer-aided detection does not show evidence of benefits in the real world [20]. FP reduction is a critical issue in AI lung nodule detection. In our current study, our AI-assisted model can greatly reduce the FP rate and improve the performance of doctors.

\section{Limitations of the study}

Our AI-assisted model improved the accuracy of reading $\mathrm{CT}$ scans and the work efficiency of doctors. However, this study has some limitations. First, this research focused on the detection of lung nodules, but 
did not focus on the differentiation of benign and malignant nodules. We did not analyze the performance of the lung cancer using the current model. Therefore, such an assistant system requires further research to confirm the diagnosis of lung cancers. However, through early detection of small lung nodules, we believe that early detection of lung cancer is still helpful. Second, the lack of a gold standard is a common problem in the AI detection of lung nodules [10]. Biopsy of lung nodules can confirm the correctness, but this is not feasible in most cases. In this study, we used the consensus of most experts as the reference standard. This approach was similar to that of previous studies on AI detection of lung nodules [10]. Third, the incidence of lung nodules varies with different characteristics of the study population, such as race, age, and smoking status. Therefore, the accuracy of AI differs for different populations $[10,20]$. Our current system still needs to be used in other ethnic groups.

\section{Conclusions}

Missed lung cancer has potentially serious medicolegal implications for doctors. The reasons for misdiagnosis on CT scans are related to specific characteristics of the undetected lesion, such as small size, ground-glass appearance, and central location. Manual detection with a large number of CT scans is a great burden, requires attention, is very timeconsuming for doctors, and is prone to errors. In our study, AI assistant programs decreased the incidence of misinterpretation of lung nodules in the errorprone characteristics of lung nodules.

\section{Acknowledgements}

This study was supported by grants from the Taipei Tzu Chi Hospital and Buddhist Tzu Chi Medical Foundation (TCRD-TPE-109-24(2/3)).

\section{Competing Interests}

The authors have declared that no competing interest exists.

\section{References}

1. Liu J, Cao L, Akin O, et al. Accurate and robust pulmonary nodule detection by $3 \mathrm{D}$ feature pyramid network with self-supervised feature learning. arXiv. 2019; 1907:11704.

2. Dziedzic R, Marjański T, Rzyman W. A narrative review of invasive diagnostics and treatment of early lung cancer. Transl Lung Cancer R. 2021; 10(2):1110-23.

3. Morozov SP, Gombolevskiy VA, Elizarov AB, et al. A simplified cluster model and a tool adapted for collaborative labeling of lung cancer CT scans. Comput Meth Prog Bio. 2021; 206:106111.

4. Chikontwe P, Kim M, Nam SJ, et al. Multiple instance learning with center embeddings for histopathology classification. MICCAI. 2020.

5. Ilse M, Tomczak J, Welling M. Attention-based deep multiple instance learning. ICML. 2018.

6. Sadafi A, Makhro A, Bogdanova A, et al. Attention based multiple instance learning for classification of blood cell disorders. MICCAI. 2020;1-11.
7. Xu G, Song Z, Sun Z, et al. Camel: A weakly supervised learning framework for histopathology image segmentation. IEEE/CVF International Conference on Computer Vision (ICCV). 2019;10681-90.

8. Bilen H, Vedaldi A. Weakly supervised deep detection networks. CVPR. 2019;2846-54.

9. Hancock MC, Magnan JF. Lung nodule malignancy classification using only radiologist-quantified image features as inputs to statistical learning algorithms: probing the Lung Image Database Consortium dataset with two statistical learning methods. J Med Imaging (Bellingham). 2016; 3(4):044504.

10. Cui S, Ming S, Lin Y, et al. Development and clinical application of deep learning model for lung nodules screening on CT images. Sci Rep. 2020; 10(1):13657.

11. Del Ciello A, Franchi P, Contegiacomo A, et al. Missed lung cancer: when, where, and why? Diagn Interv Radiol. 2017; 23(2):118-26.

12. Zhao Y, de Bock GH, Vliegenthart R, et al. Performance of computer-aided detection of pulmonary nodules in low-dose CT: comparison with double reading by nodule volume. Eur Radiol. 2012;22(10):2076-84.

13. Devaraj A. Missed cancers in lung cancer screening--more than meets the eye. Eur Radiol. 2015; 25(1):89-91.

14. Li F, Sone $\mathrm{S}$, Abe H, et al. Lung cancers missed at low-dose helical CT screening in a general population: comparison of clinical, histopathologic, and imaging findings. Radiology. 2002; 225(3):673-83.

15. Benzakoun J, Bommart S, Coste J, et al. Computer-aided diagnosis (CAD) of subsolid nodules: Evaluation of a commercial CAD system. Eur J Radiol. 2016; 85(10):1728-34.

16. Ali I, Hart GR, Gunabushanam G, et al. Lung Nodule Detection via Deep Reinforcement Learning. Front Oncol. 2018; 8:108.

17. Cao H, Liu H, Song E, et al. A Two-Stage Convolutional Neural Networks for Lung Nodule Detection. IEEE J Biomed Health Inform. 2020; 24(7):2006-15.

18. Dou Q, Chen H, Yu L, et al. Multilevel Contextual 3-D CNNs for False Positive Reduction in Pulmonary Nodule Detection. IEEE Trans Biomed Eng. 2017; 64(7):1558-67.

19. Setio AA, Ciompi F, Litjens G, et al. Pulmonary Nodule Detection in CT Images: False Positive Reduction Using Multi-View Convolutional Networks. Ieee T Med Imaging. 2016; 35(5):1160-9.

20. Walter JE, Heuvelmans MA, Bock GH, et al. Characteristics of new solid nodules detected in incidence screening rounds of low-dose CT lung cancer screening: the NELSON study. Thorax. 2018; 73(8):741-7. 